\title{
Palabras de Aida Navas durante la celebración de los 45 años de egreso del primer grupo de terapeutas ocupacionales de la Universidad Nacional de Colombia ${ }^{1}$
}

Aida Navas ${ }^{2}$

Es un privilegio estar hoy acá celebrando el Día Nacional del Terapeuta Ocupacional y la conmemoración de los 45 años del primer grupo de terapeutas ocupacionales colombianos egresados de esta Universidad.

Agradezco el honor que me han hecho los docentes organizadores para dirigir unas palabras.

Lo primero es dar un profundo agradecimiento a todas nuestras profesoras y maestras, las de ayer, las de hoy y las de siempre. Un reconocimiento especial para Patricia Lang de Pardo y Alicia Trujillo Rojas como iniciadoras de la formación de terapeutas ocupacionales en Colombia y en la Universidad.

También recordar a las pioneras de nuestra carrera, a todas y cada una de las que siguieron su proyecto creando servicios de Terapia Ocupacional, formándose en la docencia y generan- do las bases del futuro desarrollo. No quiero omitir nombres, a todas un especial reconocimiento.

El proceso que ha tenido la carrera en la Universidad Nacional es un largo, permanente, continuo y fructífero trabajo en la identificación de las bases y las teorías científicas, procedimentales, humanistas y ejecutivas de la profesión.

45 años dan cuenta de una prolífica producción en avances curriculares académicos, de investigación, de propuestas y definiciones en la construcción de conocimiento y conceptualización de la Terapia Ocupacional, de apoyo y propuestas en la generación de políticas públicas, aportes importantes al desarrollo de las personas, las comunidades y el país en el marco del objeto de conocimiento de la profesión y de los objetivos misionales de la propia Universidad.

\footnotetext{
${ }^{1}$ Palabras compartidas el 17 de noviembre de 2011 en la sala anexa del Auditorio León de Greiff de la Universidad Nacional de Colombia en Bogotá, en el marco de la celebración del Día Nacional del Terapeuta Ocupacional y los 45 años de egreso de la primera promoción del terapeutas ocupacionales de la Universidad Nacional de Colombia.

2 Terapeuta Ocupacional egresada de la Universidad Nacional de Colombia, primero en julio de 1973, con el título de Técnica en Terapia Ocupacional, y posteriormente en noviembre de 1977, con el de Licenciada en Terapia Ocupacional. Al momento de este evento ejercía como presidenta del Consejo Directivo Nacional del Colegio Colombiano de Terapia Ocupacional. Durante más de 40 años, la T.O. Aida Navas ha sido miembro activa de la Asociación, ahora Colegio, donde ha ocupado diferentes cargos en el Consejo Directivo.
} 
Ser Universidad Nacional es una impronta indeleble a la que no se puede ser inferior y que compromete el ser, el pensar y el actuar... y la emoción

El "somos Universidad Nacional" nos identifica, nos convoca, nos agrupa, nos fortalece y nos engrandece.

No resisto la tentación de recordar que nuestra promoción de julio de 1973 inició un movimiento para que la Universidad revisara el pensum de nuestras carreras (en ese momento Terapia Física, Terapia del Lenguaje y Terapia Ocupacional) para elevar los estándares de formación y poner la carrera al nivel que le correspondía. Si mal no recuerdo resistimos un mes diciendo que no nos graduábamos hasta que no hubiera un compromiso formal de las directivas, tanto de la Facultad como de la Universidad. No lo logramos en ese momento, pero si se generó un análisis, y ya en 1976, los profesionales egresados hasta ese año hicimos todo el proceso reglamentario para la profesionalización. Desde ese momento, a partir de un currículo construido con rigor y validez académica, el desarrollo del programa ha sido positivo.

Nos movíamos mucho. A comienzos de 1973 peleábamos en la Facultad contra ASCOFAME por propuestas de reforma a los pensum de salud generados desde universidades extranjeras y peleábamos defendiendo nuestro hospital San Juan de Dios. Recuerdo como anécdota, una marcha que salía de la $10^{\underline{a}}$ con $1 \stackrel{a}{ }$, mi compañera Cecilia Díaz, la opita, salió de primera en una foto de periódico al frente de la marcha, iy claro!, sus papás llamaron desde Neiva a preguntar iqué era lo que estaba haciendo, no que estaba era estudiando? Ese día había estudiantes de diferentes universidades, sorprendentemente ya empezaban a manifestarse de la Javeriana y el Rosario con sus blusas blancas. Cuando se alborotó el asunto y llegaron las jaulas a sitiarnos, todo el mundo se quito las blusas y a correr. Nosotras no podíamos, hay que recordar el uniforme, así que solo corrimos. Muchos se refugiaron en el hospital, a mi casi me golpea un policía, corrí por el Policarpa Salavarrieta, que en ese momento era un barrio de invasión, hacia la Avenida Caracas, y me metí escondida en una buseta. Mis compañeras pensaban que yo estaba en la cárcel y ellas pasaron la noche sitiadas en San Juan.

Yo no sé si eso para mí desde ya era parte del compromiso político naciente, o como dice Frank Kronenberg, el bicho de la política.

Pasando a lo actual, quiero pensar con ustedes en los paradigmas y propuestas de la Terapia ocupacional de hoy, que nos plantean responsabilidades; y en especial referirme a lo que Kronenberg identifica como la responsabilidad de "las tres $\mathrm{P}$ ": personal, profesional y política. Estamos frente a un panorama disciplinar que abarca y transciende cada acción que tengamos en el ejercicio profesional.

Como lo plantean los nuevos constructos desde Latinoamérica, los Terapeutas ocupacionales no podemos sustraernos al impacto de los determinantes sociopolíticos, económicos, estructurales, históricos y poblacionales, a las realidades y dinámicas sociales, y 
por supuesto, a la capacidad transformadora que nos es posible construir de manera creativa, integrada, ética con cada persona en su especial y particular entorno.

Sandra Galheigo nos dijo en el Congreso Mundial de Chile 2010 que: "Al compartir las experiencias y nuevas narrativas se nos ofrece la oportunidad de interrogar los puntos de vista hegemónicos de la Terapia Ocupacional y crear nuevas oportunidades para la profesión".

La misma profesora señala que: "La creación y el uso del conocimiento es compleja y no sucede automáticamente, y en vista de ello, debemos evitar un enfoque sobre la creación del conocimiento que se aplique a escala universal sin considerar cultura, contexto y necesidades".

En esta perspectiva es que resulta especialmente revelador y motivador el texto lanzado hace cerca de dos meses por el Departamento y el Grupo de Investigación Ocupación y Realización Humana, en el que analiza la Ocupación Humana en perspectiva filosófica, social y evolutiva, y en donde se estudia y se reflexiona la ocupación como proceso subjetivante, sociocultural y ecológico.

Yo personalmente percibo el poderoso aliento que tiene muestra profesión y los paradigmas que tiene por delante en la confluencia de sus construcciones teóricas y científicas de base, los evidentes y positivos resultados que ha tenido y sigue teniendo en el ejercicio profesional de estos modelos, y de otro lado, las exigencias que el contexto sociocultural, económico y político nos hace en tanto ciudadanos profesionales cuyo objeto de conocimiento es la ocupación y su significación en personas y comunidades en condiciones de indefensión, vulnerabilidad o simplemente realidades de vida.

La realidad política, social y de dinámica poblacional de Colombia nos sitúa en una condición paradójica, entre lo que es nuestro deber ser, en virtud de los avances conceptuales, científicos y tecnológicos de la Terapia ocupacional y la Ciencia de la Ocupación Humana en el mundo, y lo que tenemos como el posible poder ser, debido a las limitaciones de orden político, económico, reglamentario e incluso de paradigmas sociales no suficientemente aclarados.

Encontramos de un lado las difíciles e inequitativas condiciones de vida de la mayoría de la población en términos de determinantes de la salud: seguridad alimentaria, vivienda, agua potable, servicios de salud efectivos y accesibles, educación y esparcimiento; las situaciones de enfermedad partiendo del recién nacido sano o en riesgo; los avances de la ciencia para permitir la vida a recién nacidos con Apgar que hace un tiempo habrían sido imposibles, pero que requieren intervenciones permanentes y seguras para garantizar la viabilidad de su neurodesarrollo; las secuelas de diferentes patologías en niños y niñas; la importante incidencia de las condiciones de sedentarismo, abandono físico y emocional, parcial o total, por parte de los padres; las nuevas dinámicas en los procesos de aprendizaje; las preocupantes secuelas de violencia, tanto del conflicto armado, como de 
la violencia social y familiar; las secuelas por patologías cardiovasculares, neurológicas, sistémicas, de trauma y emocionales; las cada vez más evidentes condiciones de fatiga laboral con sus consecuencias de ansiedad, agotamiento y depresión; los retos que imponen la protección de los trabajadores, el aumento en la expectativa de vida de la población, y en lo colectivo, la demanda creciente surgida desde poblaciones vulnerables en diferentes contextos y con grados diferentes de complejidad, para la paliación, remediación y transformación de sus condiciones y expectativas de vida.

El manejo que de estas características de vida de la población, hace la normatividad nacional e internacional, supone de nuestra parte el conocimiento de la misma y la generación de acciones políticas, administrativas, investigativas, académicas y profesionales como forma de incidir en los contextos social, económico y ético. En lo individual y en lo colectivo.

Imagino que cuando he mencionado cada uno de estos escenarios, ustedes habrán identificado algo por hacer. Y posiblemente se me ha quedado algo en el tintero.

De otro lado, nos encontramos con un panorama estadístico y básicamente con un panorama real de complejidad en la condiciones laborales y de indicadores que aparentemente señalan, como lo ha pretendido mostrar el Ministerio de Protección Social, la poca pertinencia de una profesión que muestra una tasa de desempleo del $47 \%$ aproximadamente. $Y$ cuando hablamos de empleo, tenemos que referirnos a la posibilidad de empleo en condiciones de adecuada remuneración, de estabilidad y garantía de seguridad social, tal como lo establece la Constitución.

Nos encontramos con que en la Clasificación Nacional de Ocupaciones aparecemos en el área ocupacional 31 (nivel de preparación A), numeral 314, como profesionales en terapia y valoración. No estamos identificados como profesión, a pesar de la promulgación desde 2005 de la Ley 949.

El estudio del Ministerio de la Protección Social y el Centro de Proyectos para el Desarrollo - Cendex, de la Universidad Javeriana, sobre recursos humanos de la salud en Colombia, publicado en el 2008 en su tercera edición, señala por ejemplo que: "el stock de profesionales en terapias resulta superior a la estimación de demanda requerida bajo el primer escenario, lo cual indica que, asumiendo estabilidad en el nivel de aseguramiento de 2006, el total de profesionales que saldrán al mercado laboral será superior a la demanda requerida durante el período 2007-2011. Sin embargo, al asumir lapsos de 5 a 10 años para el cubrimiento universal de salud, la demanda de profesionales en terapias requerida por el mercado superará el stock estimado para el mismo período". Es decir, que cuando toda la población pueda tener salud como derecho fundamental, será equilibrada la demanda de terapeutas ocupacionales.

El mismo estudio señala que en el perfil ocupacional de los terapeutas ocupacionales se evidencia una mayor fle- 
xibilidad y polifuncionalidad que para otras, como ellos denominan, "terapias". El diagnóstico y tratamiento de la enfermedad y la atención de pacientes tiene una participación de 31.99\% de los profesionales; apoyo administrativo de servicios, un $11.09 \%$; educación en salud, 1.1\%; mantenimiento del bienestar del paciente, $0.70 \%$, formación, diseño y programación de servicios, 0.16\%; planeación en salud, $0.03 \%$, entre otros ítems analizados.

Estos datos presentados de forma rápida, nos pueden llevar a diferentes análisis.

¿Cuál creemos que es la razón para que con altos indicadores de orden epidemiológico de enfermedad, de condiciones de discapacidad física, sensorial, mental, con comunidades urbanas y rurales que requieren apoyo profesional y acompañamiento, nuestros profesionales, que tienen las competencias profesionales en todos estos campos del saber y del actuar, no encuentren escenarios laborales que den respuesta a la demanda de servicios de la población? Uno de los análisis que yo he hecho es que muy posiblemente, y más allá de contextos externos de desempleo del país y que escapan a un análisis particular, la tasa de desempleo que aduce el estudio y algunos argumentos de funcionarios del Ministerio para desestimar la pertinencia de la formación de terapeutas ocupacionales, no es coherente con los mismos indicadores de prevalencia de enfermedades y condiciones de salud y calidad de vida que caen en la órbita del nuestro ejercicio profesional. La lectura que puede hacerse es que el sistema actual no permite, no garantiza que la población pueda acceder en condiciones de equidad y universalidad, ni que los profesionales tengan oportunidades de empleo digno y estable.

Pienso que la responsabilidad profesional y política tiene en estos espacios un trabajo que requiere mucha más dinámica y cohesión. El Colegio ${ }^{3}$ ha tenido en toda su historia participación en escenarios gubernamentales, políticos, institucionales públicos y privados. En estos últimos años hemos hecho presencia y continuamos haciéndolo. Hablar en los Grupos de Enlace Sectorial del Ministerio de Protección Social; en el recinto del Congreso sobre Talento Humano en Salud; con la CRES (Comisión de Regulación de Salud) de modificación de los planes de beneficio de la población y de las inequidades y faltas de oportunidad en el acceso a servicios de Terapia Ocupacional; de los aportes de Terapia ocupacional en tecnología en salud para la población; de la garantías de nuestros profesionales en ámbitos laborales, educativos, de elaboración de tecnología para rehabilitación de miembros superiores; trabajar en la definición de competencias profesionales, fijando de paso una posición gremial en lo que debe representar para el país el conocimiento; revisar y dar concepto al Congreso de la República en un proyecto de Ley de Salud Mental; hablar con el Viceminis-

\footnotetext{
${ }^{3}$ Nota de la editora: Refiriéndose al Colegio Colombiano de Terapia Ocupacional.
} 
terio de Educación Superior para que el término Terapias sea desagregado identificando cada profesión; estos y muchos otros hacen parte del trabajo que se hace en el Colegio en defensa de la disciplina, en beneficio de los profesionales, pero básicamente aportando a la construcción de espacios para las personas y las comunidades, en la construcción y ojalá transformación de país.

Por otro lado, ¿cuál es la razón para que nuestras universidades vean disminuir sensiblemente el número de estudiantes de bachillerato que consideran como opción de vida profesional la Terapia Ocupacional?

Posiblemente lo que se planteó anteriormente tenga incidencia. Es seguro que los programas de formación tienen su propia visión del fenómeno. Sin embargo, hay un tema que de vez en cuando retomamos y analizamos y es el del reconocimiento social.

En un texto del sociólogo Amando de Miguel, español, sobre la aportación de los colegios profesionales a la sociedad, que en Colombia corresponderían a las sociedades y asociaciones científicas, señala que: "En enero de 2003 se publicaba el barómetro de opinión de la ciudadanía respecto a los abogados. De él se desprende la aceptación del abogado por la sociedad, ya que es percibido como alguien en quien se debe poder confiar plenamente.También existe el factor de "entrega absoluta". En esa prospección aparece que una gran parte de personas entienden que la tarea del abogado no es tanto defender lo justo como aquello que beneficia a su cliente. Se muestra, además, la existencia de una doble pauta valorativa, coexistiendo una imagen global y genérica de la abogacía, y una imagen más específica e individualizada del abogado. Esa última arroja una valoración ampliamente positiva. La abogacía, globalmente considerada, es objeto de matices, rasgos y valoraciones positivas, junto con percepciones fuertemente negativas. Muchas veces esas visiones son expresivas de tópicos y estereotipos ampliamente arraigados en la conciencia social.

El mencionado barómetro resulta muy adecuado para saber en cada momento qué posición se ocupa, como profesión, en la sociedad.

Considera el sociólogo además que los Colegios Profesionales forman parte sustancial de lo que se llama "sociedad civil", porque ejercen funciones de interés general sin que dependan jerárquicamente del aparato público. Es decir, no los pagan los contribuyentes.

Respecto a este concepto de Sociedad civil, el Doctor Israel Cruz, profesor de la Universidad del Rosario, mencionó en una reciente presentación algo que encuentro ajustado a la realidad y pertinente a estas reflexiones: que los Estados dialogan con la Sociedad Civil organizada.

Es en este punto, retomando el barómetro, donde creo que tenemos que recapitular y generar acciones que nos permitan salir un poco de la situación de paradoja de nuestra profesión. Poseemos una importante base científica y conceptual, avances en investigación y desarrollo del ejercicio del orden na- 
cional y mundial, perspectivas de desempeño en diferentes escenarios de la vida nacional, pero con demasiada frecuencia sentimos la dificultad en el reconocimiento social, en la identificación de una pauta valorativa del terapeuta ocupacional y su accionar.

En tanto logremos un ejercicio profesional riguroso, efectivo y ético y una cohesión como sociedad civil organizada de terapeutas ocupacionales, la misma sociedad tendrá una imagen más específica del terapeuta y una imagen global y genérica de la profesión. Para los estudiantes que inician su formación, debería ser interesante ingresar a ese cuerpo profesional del cual identifica sus objetivos y sus logros.

Para muchos profesionales esta participación organizada resulta innecesaria y espera retribuciones casi siempre muy concretas. Y para los recién egresados, no hace parte de las prioridades. Tengo muy claro que no es una dificultad exclusiva de nuestro gremio, pero en el trabajo que vengo desarrollando desde hace más de 6 años, compartiendo con diferentes disciplinas, si puedo asegurarles que nuestra situación es especialmente preocupante. Estoy convencida de la necesidad de superar errores, diferencias, dificultades, lo que sea que nos impide sentir pertenencia por el gremio y lo que representa. Cuando el Estado, la sociedad y la población, como lo hemos visto, nos demandan acciones, definiciones, participaciones, creo que el consenso amplio y en lo posible mayoritario en torno a lo que es y representa la Terapia Ocupacional, resulta necesario y pertinente. Dejo a ustedes la valoración de lo que he planteado, pero estoy convencida, necesitamos organizarnos más y mejor. Necesitamos pertenecernos.

Finalmente quiero compartir con ustedes nuestra nueva imagen y razón social: el Colegio Colombiano de Terapia Ocupacional. ${ }^{4}$

Gracias.

\footnotetext{
${ }^{4}$ En 2011, la hasta entonces Asociación Colombiana de Terapia Ocupacional modifica estatutariamente su razón social para llamarse Colegio Colombiano de Terapia Ocupacional, cambio que obedece a la normatividad establecida en la ley 1164 de 2007 que regula el Talento Humano en Salud. Dicho cambio de denominación implicó la modificación de su imagen a la que hoy en día identifica al Colegio.
} 